\title{
Adders for the patterned starter in some non-abelian groups
}

\section{Philip A. Leonard}

Starters with adders in abelian groups of odd order have been used extensively in the construction and study of Room squares. It is possible to define these concepts in non-abelian groups, with similar applicability to Room squares. Examples are given of adders for the "patterned starter" in the non-abelian groups of order $p q, p$ and $q$ primes larger than $3, p \equiv q \equiv 3$ $(\bmod 4), q \equiv 1(\bmod p)$.

One fruitful approach to the construction and study of Room squares has been that based on starters and adders in groups of odd order (see [2]).

If $G$ is a multiplicative group of order $2 n+1$, and $H=G \backslash\{1\}$, a starter in $G$ is a set $X=\left\{\left(x_{i}, y_{i}\right) \mid 1 \leq i \leq n\right\}$ of ordered pairs such that

$$
H=\left\{x_{i}, y_{i} \mid 1 \leq i \leq n\right\}=\left\{x_{i} y_{i}^{-1}, y_{i} x_{i}^{-1} \mid 1 \leq i \leq n\right\} .
$$

If $X$ is a starter in $G$, an adder for $X$ is an ordered set $A=\left(a_{1}, \ldots, a_{n}\right)$ of distinct elements of $H$ such that

$$
H=\left\{x_{i} a_{i}, y_{i} a_{i} \mid 1 \leq i \leq n\right\}
$$

Given a starter $X$ with adder $A_{X}$, a Room square of side $2 n+1$ may be constructed as follows: let $R$ be a square array, with rows and

Received 4 December 1973. 
and columns indexed by the elements of $G$. The cell in position $(g, h)$ is empty unless $h=a_{i} g$ for some $i$, and in this case it contains the pair $\left\{x_{i} a_{i} g, y_{i} a_{i} g\right\}$.

This note contains examples of starters with adders in certain nonabelian groups (see [2], Problem 9).

Let $p$ and $q$ be odd primes, larger than 3 , with $p \equiv q \equiv 3$ $(\bmod 4)$ and $q \equiv I(\bmod p)$, and let $G$ be the non-abelian group of order $p q$ defined by $a^{p}=1, b^{q}=1, a^{-1} b a=b^{r}$, where $r$ is an integer of order $p$ (modulo $q$ ) (see [1]). Products in $G$ are given by

$$
a^{x} b^{y} \cdot a^{u} b^{v}=a^{x+u_{b} y r^{u}+v}, 0 \leq x, u \leq p-1,0 \leq y, v \leq q-1 .
$$

Let $R_{p}$ (respectively $N_{p}$ ) denote the set of quadratic residues (respectively non-residues) modulo $p$, with $R_{q}$ and $N_{q}$ defined similarly. Let

$$
S=\left\{g=a^{x} b^{y} \mid x \in R_{p} \text {, or, } x=0 \text { and } y \in R_{q}\right\} \text {. }
$$

Since $p \equiv q \equiv 3(\bmod 4)$, we have the following

LEMMA. $X=\left\{\left(g, g^{-1}\right) \mid g \in s\right\}$ is a starter in $G$.

The starter $X$ of the lemma is called the patterned starter in $G$. Examples of adders for $X$ are given in the following result. The proof, which is a routine matter of verification, is omitted.

THEOREM. For $m \in N_{p} \backslash\{-1\}$ and $n \in N_{q} \backslash\{-1\}$, define $k$ by $k \equiv \frac{m+1}{m-1}$ $(\bmod p)$, and $\tau$ by $\tau \equiv \frac{n+1}{n-1}(\bmod q)$. Then $A_{X}=\left(h_{g}\right), g \in S$, with $h_{g}=a^{k x_{b} l y}$ for $g=a^{x} b^{y}$, is an adder for the patterned starter $X$, provided $\imath^{p} \neq \pm(\bmod q)$.

For example, with $p=7$ and $q=67$, we may take $m=n=3$, giving $k=\tau=2$, which is suitable as $2^{7} \equiv 61(\bmod 67)$. For the smallest eligible values of $p$ and $q$, namely $p=7$ and $q=43$, $\tau=2$ is not admissible, but $l=3$ (corresponding to $n=2$ ) is, as 
$3^{7} \equiv 36(\bmod 43)$

\section{References}

[1] Marshall Hall, Jr, The theory of groups (The Macmillan Company, New York, 1959).

[2] W.D. Wallis, "Room squares", Combinatorics: Room squares, sum-free sets, Hadomard matrices, 33-121 (Lecture Notes in Mathematics, 292. Springer-Verlag, Berlin, Heidelberg, New York, 1972).

Department of Mathematics,

Arizona State University,

Tempe,

Arizona,

USA. 